\title{
Heterogeneity of glucagonomas due to differential processing of proglucagon-derived peptides
}

\author{
Benjamin G Challis ${ }^{1,2, *}$, Nicolai J Wewer Albrechtsen ${ }^{3,4,}$, Vishakha Bansiya ${ }^{2}$, \\ Keith Burling', Peter Barker', Bolette Hartmann ${ }^{3,4}$, Fiona Gribble ${ }^{1}$, Stephen O’Rahilly ${ }^{1,2}$, \\ Jens $\mathrm{J} \mathrm{Holst}^{3,4}$ and Helen L Simpson ${ }^{2}$ \\ ${ }^{1}$ Wellcome Trust-MRC Institute of Metabolic Science, University of Cambridge, Cambridge CB2 OQQ, UK \\ ${ }^{2}$ Wolfson Diabetes and Endocrinology Clinic, Institute of Metabolic Science, Cambridge University Hospitals NHS \\ Foundation Trust, Addenbrookes Hospital, Box 281, Cambridge CB2 0QQ, UK \\ ${ }^{3}$ Department of Biomedical Sciences, Faculty of Health and Medical Sciences and ${ }^{4}$ Faculty of Health and \\ Medical Sciences, Novo Nordisk Foundation Center for Basic Metabolic Research, University of Copenhagen, \\ Blegdamsvej 3B, Copenhagen DK-2200, Denmark \\ *(B G Challis and N J W Albrechtsen contributed equally to this work)
}

\section{Summary}

Pancreatic neuroendocrine tumours (pNETs) secreting proglucagon are associated with phenotypic heterogeneity. Here, we describe two patients with pNETs and varied clinical phenotypes due to differential processing and secretion of proglucagonderived peptides (PGDPs). Case 1, a 57-year-old woman presented with necrolytic migratory erythema, anorexia, constipation and hyperinsulinaemic hypoglycaemia. She was found to have a grade 1 pNET, small bowel mucosal thickening and hyperglucagonaemia. Somatostatin analogue (SSA) therapy improved appetite, abolished hypoglycaemia and improved the rash. Case 2, a 48-year-old male presented with diabetes mellitus, diarrhoea, weight loss, nausea, vomiting and perineal rash due to a grade 1 metastatic PNET and hyperglucagonaemia. In both cases, plasma levels of all measured PGDPs were elevated and attenuated following SSA therapy. In case 1, there was increased production of intact glucagon-like peptide 1 (GLP-1) and GLP-2, similar to that of the enteroendocrine L cell. In case 2, pancreatic glucagon was elevated due to a pancreatic $\alpha$-cell-like proglucagon processing profile. In summary, we describe two patients with pNETs and heterogeneous clinical phenotypes due to differential processing and secretion of PGDPs. This is the first description of a patient with symptomatic hyperinsulinaemic hypoglycaemia and marked gastrointestinal dysfunction due to, in part, a proglucagon-expressing PNET.

\section{Learning points:}

- PGDPs exhibit a diverse range of biological activities including critical roles in glucose and amino acid metabolism, energy homeostasis and gastrointestinal physiology.

- The clinical manifestations of proglucagon-expressing tumours may exhibit marked phenotypic variation due to the biochemical heterogeneity of their secreted peptide repertoire.

- Specific and precise biochemical assessment of individuals with proglucagon-expressing tumours may provide opportunities for improved diagnosis and clinical management. 


\section{Background}

Pancreatic neuroendocrine tumours (pNETs) that secrete glucagon are associated with well-recognised clinical features of the glucagonoma syndrome including necrolytic migratory erythema (NME), weight loss, diarrhoea, anaemia, stomatitis, thromboembolic complications and sometimes diabetes mellitus. Less well-recognised are the paraneoplastic phenomena associated with tumoural secretion of proglucagon-derived peptides (PGDPs) including marked gastrointestinal dysfunction and hyperinsulinism. Here, we describe two subjects with proglucagon-expressing pNETs with marked phenotypic heterogeneity. Case 1 represents the first report of a patient presenting with both symptomatic hypoglycaemia associated with excess proinsulin and insulin as well as gastrointestinal dysfunction due, in part, to tumoural overexpression of PGDPs. Case 2 typifies tumoural glucagon secretion. In both cases, plasma levels of PGDPs were evaluated by well-characterised specific immunoassays and chromatographic methods before and after treatment with somatostatin analogue (SSA). Together these cases expand the clinical spectrum associated with proglucagonexpressing tumours and illustrate, through detailed biochemical characterisation, opportunities for biochemical sub-classification and improved understanding of the clinical manifestations associated with these rare tumours.

\section{Case presentation}

\section{Case 1}

A 57-year-old female presented with a 12-month history of a painful and blistering eczematous rash involving her limbs and face and a number of concurrent symptoms including constipation, early satiety, bloating, nausea and vomiting and weight loss. She reported symptoms consistent with hypoglycaemia and although paramedic assistance was sought on several occasions prior to presentation, a severe needle phobia precluded assessment of blood glucose levels outside of hospital. On examination she was pale and emaciated (BMI $16.5 \mathrm{~kg} / \mathrm{m}^{2}$ ) with an extensive eczematous rash involving all limbs, sacrum and face (Fig. 1).

\section{Case 2}

A 48-year-old male with newly diagnosed diabetes mellitus presented with abdominal pain, diarrhoea, nausea and weight loss. He had a longstanding history of

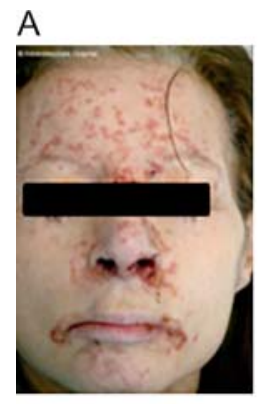

Pre-octreotide

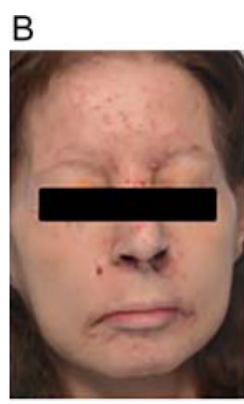

Day 4

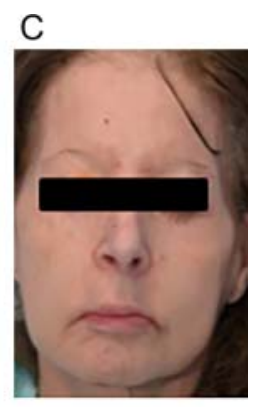

Day 21

\section{Figure 1}

Photographs of case 1 showing (A) necrolytic migratory erythema before treatment with octreotide $(100 \mu \mathrm{g}$ thrice daily) and progressive resolution following 4 days (B) and 21 days (C) of octreotide therapy.

a recurrent perineal skin rash which relapsed frequently despite repeated courses of topical glucocorticoid and antifungal preparations. On examination he was tachycardic (120 beats/min), hypotensive $(85 / 60 \mathrm{mmHg})$ with a BMI of $24 \mathrm{~kg} / \mathrm{m}^{2}$. He had hepato-splenomegaly, gross generalised oedema and eczematous perineal rash.

\section{Investigation}

\section{Case 1}

Laboratory investigations revealed normocytic anaemia (Hb $70 \mathrm{~g} / \mathrm{l}$ (normal range (NR): 115-160) and mean corpuscular volume (MCV) $85.2 \mathrm{fl}$ (NR: 80-100)), hypoalbuminaemia (21 g/l (NR: 30-51)), pancytopaenia, hypoglycaemia $(2.8 \mathrm{mmol} / \mathrm{l})$ and low zinc $(9 \mu \mathrm{mol} / \mathrm{l}$; NR: 12-23). HbA1c ( $45 \mathrm{mmol} / \mathrm{mol}$; NR: 30-45), corrected calcium (2.32 mmol/1; NR: 2.1-2.5) and biochemical indices of renal and hepatic function were normal. Differential diagnoses guiding further investigation included malignancy, malabsorption or possible eating disorder.

Computed tomography (CT) revealed a $3 \times 2 \mathrm{~cm}$ pancreatic mass with widespread liver metastases, multiple pulmonary emboli and, unexpectedly, marked mucosal thickening and hypertrophy of the small intestine (Fig. 2A). Histology following biopsy of a hepatic metastasis demonstrated a well-differentiated grade 1 NET (MIB1, 1-2\%). Immunohistochemical analysis demonstrated staining with antibodies directed against CK19, CK5/6, synaptophysin and chromogranin, with weakly positive staining for glucagon and negative insulin immunoreactivity (Figs 1D and 2C). ${ }^{111}$ In octreotide scan with SPECT demonstrated increased uptake by the 
A

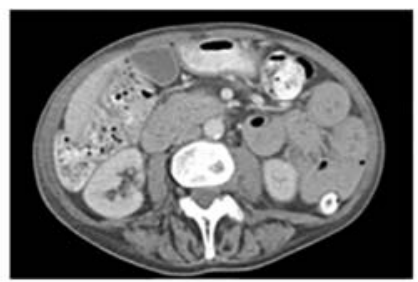

\section{B}

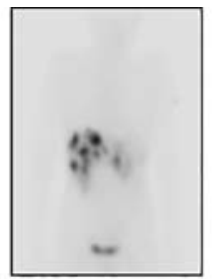

C

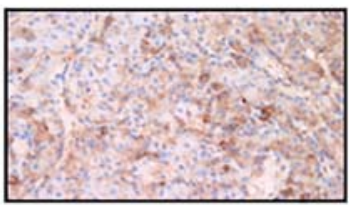

Glucagon

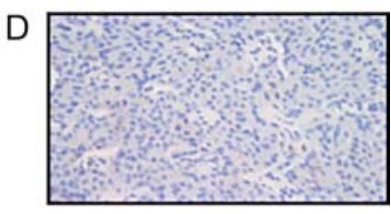

Insulin
Figure 2

(A) Computed tomography of the abdomen demonstrating thickened small bowl. (B) Somatostatin receptor scintigraphy demonstrating multiple hepatic metastases. Immunohistochemical analysis of a biopsied liver metastasis showing positive staining for (C) glucagon and negative staining for (D) insulin.

pancreatic mass and multiple liver lesions confirming octreotide avid disease (Fig. 2B).

Following hospital admission, the patient was noted to have recurrent episodes of spontaneous hypoglycaemia, which combined with radiological and dermatological findings raised suspicion of a functional islet cell tumour co-secreting insulin and glucagon. A supervised fast with concomitant measurement of additional gut hormones (Table 1) was undertaken. Hyperinsulinaemic hypoglycaemia (blood glucose $1.7 \mathrm{mmol} / \mathrm{l}$, insulin $143 \mathrm{pM}$ (NR: 0-60) and proinsulin $452 \mathrm{pmol} / \mathrm{l}$ (NR: 0-7)) was provoked following $4 \mathrm{~h}$ of fasting. Interestingly, concomitant proglucagon levels were also elevated (Table 1). Prompted by the radiological finding of thickened small intestine, plasma citrulline, a non-protein amino acid biomarker of intestinal mucosal mass was measured and found to be normal $(2.4 \mu \mathrm{mol} / \mathrm{l})$.

A 17-fold discrepancy between glucagon levels was determined when two different glucagon-specific assays were employed; the first based on a C-terminal directed antibody that detect total glucagon (i.e. proglucagon $\mathrm{X}-61$ ), and the second, a sandwich ELISA assay that only detects intact glucagon (proglucagon 33-61). To further characterise these glucagon moieties, plasma was subjected to gel filtration chromatography. Analysis of the gel filtration profile using an immunoassay specific for the C-terminus of glucagon confirmed existence of two molecules with glucagon immunoreactivity but of differing molecular sizes resulting in different elution profiles: coefficients of distribution $\left(K_{\mathrm{d}}\right), 0.28$ and 0.72 , corresponding to proglucagon (1-61) and unlabelled glucagon calibrator, respectively (Fig. 3B). Region-specific immunoassays revealed that the majority of glucagon-like peptide 1 (GLP-1) was processed into intact biologically active forms (GLP-1 ${ }_{7-36 \mathrm{NH} 2}$ and GLP-1 ${ }_{7-37}$; Table 2 and Fig. 3A and B), thereby, supporting tumour-derived GLP-1 secretion as a possible additional mechanism driving pancreatic $\beta$-cell mediated insulin release and ensuing hypoglycaemia.

Prompted by the radiological finding of small bowel hypertrophy, plasma GLP-2 levels were measured and found to be elevated (Table 2 and Fig. 3A and B). Finally, levels of the enteroglucagon-derived peptides, oxyntomodulin and glicentin were also elevated (Table 2 and Fig. 3A).

\section{Case 2}

Laboratory investigations revealed hyperglycaemia ( $24.3 \mathrm{mmol} / \mathrm{l})$ and hypoalbuminaemia (18 g/l (NR: 30-15)) with normal renal, hepatic and haematological indices. CT demonstrated a heterogeneous pancreatic mass with large volume hepatic metastases. Histology following biopsy of the large pancreatic mass demonstrated a well-differentiated grade 1 NET (MIB1, 1\%). Immunohistochemical analysis revealed positive staining with antibodies directed against CK5/6, synaptophysin, chromogranin, S100, and CK AE1/AE3 and weakly positive staining for somatostatin. Negative immunoreactivity was observed for antibodies directed against CK19, insulin, gastrin and surprisingly, glucagon. ${ }^{111}$ In octreotide scan with SPECT revealed heterogeneous uptake by the pancreatic mass with octreotide avidity observed in some hepatic metastases. A fasting gut hormone profile revealed hyperglucagonaemia (Table 2).

Table 1 Baseline fasting gut hormone profile in cases 1 and 2.

\begin{tabular}{|c|c|c|c|}
\hline Assayed hormone & Case 1 & Case 2 & $\begin{array}{c}\text { Reference } \\
\text { range }\end{array}$ \\
\hline Proglucagon (pM) & 3504 & 786 & $10-40$ \\
\hline PYY $(\mathrm{pg} / \mathrm{ml})$ & 237 & - & $25-140$ \\
\hline$P P(p M)$ & 542 & 7085 & $0-300$ \\
\hline Somatostatin (pM) & 74 & 63 & $0-150$ \\
\hline VIP (pM) & 14 & 15 & $0-30$ \\
\hline Gastrin (pM) & 10 & 17 & $0-40$ \\
\hline Chromogranin A (pM) & 146 & 1380 & $0-60$ \\
\hline Chromogranin B (pM) & 205 & 425 & $0-150$ \\
\hline
\end{tabular}



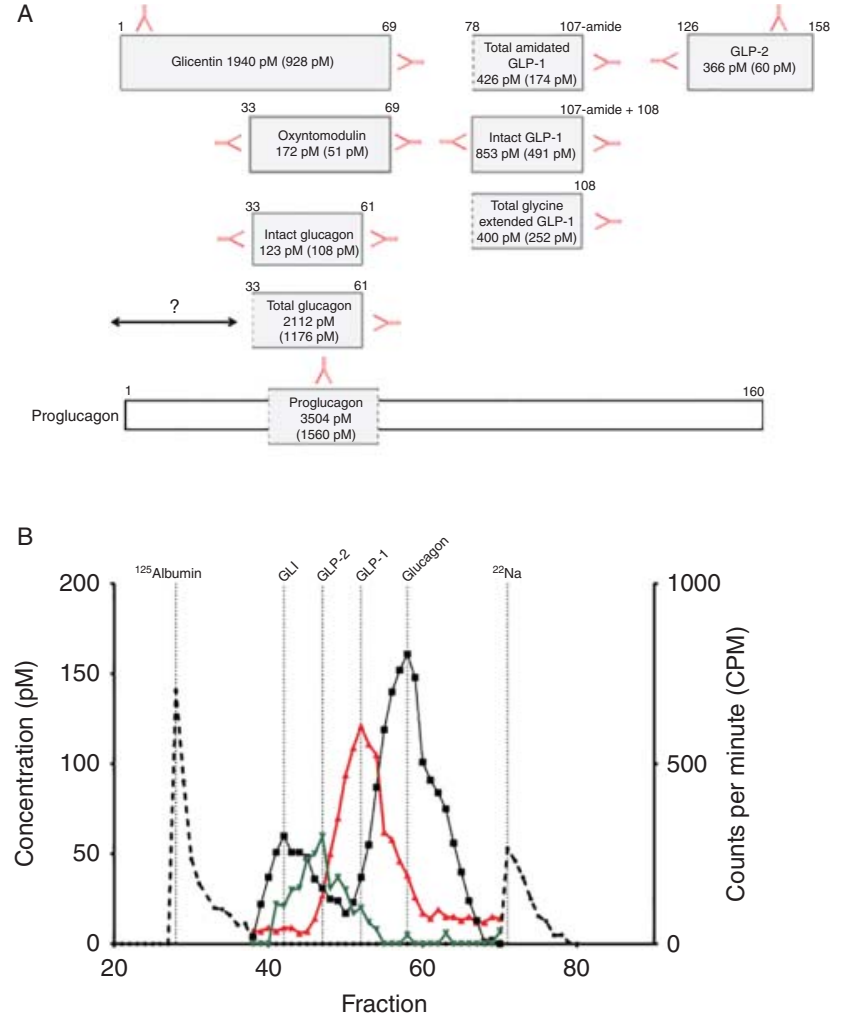

Figure 3

(A) Illustrates measured levels of proglucagon-derived peptides from case 1: glucagon, glicentin, oxyntomodulin, amidated total GLP-1, intact GLP-1, glycine-extended GLP-1 and GLP-2. Hormone levels appear as: before (after) somatostatin analogue therapy in pM. Red arrows illustrate C- or $\mathrm{N}$-terminal, or side-viewing antibodies used for measurement. '?' Refers to $\mathrm{N}$-terminal elongated glucagon. (B) Gel filtration profile of plasma from case 1. The left $y$-axis reflects hormone levels of collected fractions (100, herewith 38-70 is illustrated) using C-terminal glucagon assay (black curve), C-terminal amidated GLP-1 assay (red curve) and N-terminal GLP-2 assay (green curve). Calibrators (stippled black line) include ${ }^{125} \mathrm{I}$ albumin and ${ }^{22} \mathrm{Na}$. GLI refers to glucagon-like immunoreactivity.

Biochemically, this case had 'true' hyperglucagonaemia with similar levels of intact - and total glucagon as well as a single peak, corresponding to the glucagon calibrator, detected by gel filtration chromatography using the C-terminus assay $\left(K_{\mathrm{d}}=0.74\right.$; Fig. $4 \mathrm{~A}$ and $\left.\mathrm{B}\right)$. Interestingly, GLP-2 was higher than that observed for case 1, although, gross radiological abnormalities of the small intestine were not seen. To explore this discrepancy further, two different GLP-2 assays that recognise either the N-terminus of GLP-2 or a side-viewing assay that recognises 'total GLP-2' were employed with subsequent gel filtration chromatography. We identified one GLP-2 moiety the size of the GLP-2 calibrator $\left(K_{\mathrm{d}}=0.45\right)$ and a second, smaller, immunoreactive GLP-2 molecule $\left(K_{\mathrm{d}}=0.82\right)$, suggesting the presence of a predominant, biologically inactive GLP-2 moiety. Of the enteroglucagon-derived peptides, glicentin was elevated but not oxyntomodulin.

\section{Treatment}

\section{Case 1}

The patient was commenced on corn starch and s.c. octreotide $(100 \mu \mathrm{g}$ three times daily) resulting in an immediate improvement in appetite and abolition of hypoglycaemia. The rash dramatically improved within days of commencing treatment with typical appearances of NME becoming more apparent during the resolution phase (Fig. 1). She was commenced on Somatuline Autogel (120 mg every 28 days) with sustained clinical improvement, however died three months following her initial presentation.

\section{Case 2}

Treatment with Somatuline Autogel (120 mg once monthly) was started; however, the patient died 11 months following initial presentation.

\section{Outcome and follow-up}

No follow-up was required.

\section{Discussion}

The rare proglucagon-producing islet $\alpha$-cell tumours present with phenotypic heterogeneity due to differences in post-translational processing and peptide secretion (1). In healthy $\alpha$-cells, glucagon is the major peptide hormone

Table 2 Measured levels of proglucagon-derived peptides (pM) from cases 1 and 2 before (after) somatostatin analogue therapy.

\begin{tabular}{|c|c|c|c|}
\hline Assayed hormone & Case 1 (pM) & Case 2 (pM) & $\begin{array}{l}\text { Reference } \\
\text { range }(\mathrm{pM})\end{array}$ \\
\hline Total glucagon & $2112(1176)$ & $552(240)$ & $3-20$ \\
\hline Intact glucagon & 123 (108) & 655 (337) & $3-20$ \\
\hline Intact GLP-1 & $853(491)$ & $190(123)$ & $1-15$ \\
\hline $\begin{array}{l}\text { Total amidated } \\
\text { GLP-1 }\end{array}$ & 426 (174) & 282 (138) & $5-40$ \\
\hline $\begin{array}{l}\text { Total glycine } \\
\text { extended GLP-1 }\end{array}$ & $400(252)$ & $128(80)$ & $5-20$ \\
\hline GLP-2 & $366(60)$ & $426(138)$ & $5-50$ \\
\hline Glicentin & $1940(928)$ & $278(150)$ & $10-50$ \\
\hline Oxyntomodulin & $172(51)$ & $8(8)$ & $5-50$ \\
\hline
\end{tabular}


A

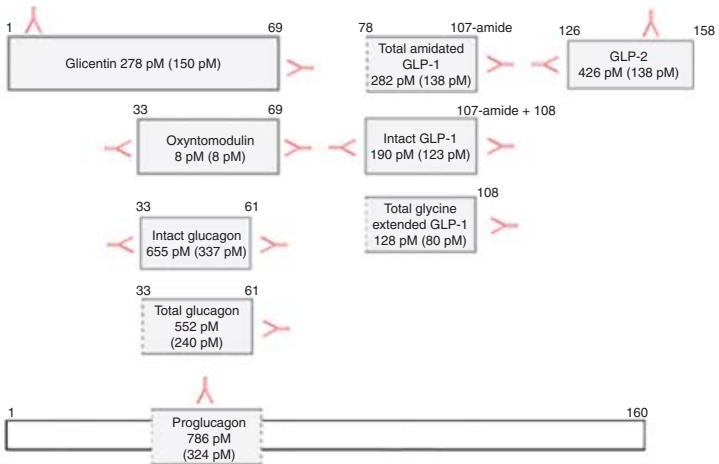

B

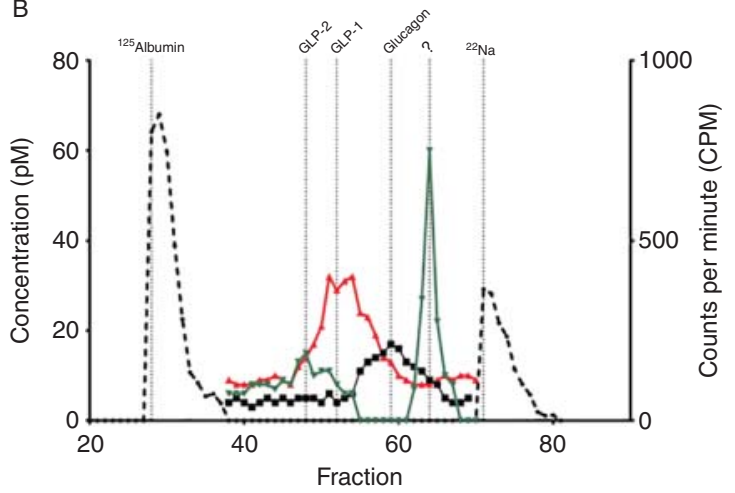

Figure 4

(A) Illustrates measured levels of different proglucagon-derived peptides from case 2: glucagon, glicentin, oxyntomodulin, amidated total GLP-1, intact GLP-1, glycine-extended GLP-1 and GLP-2. Hormone levels appear as: before (after) somatostatin analogue therapy in $\mathrm{pM}$. Red arrows illustrate C- or N-terminal, or side-viewing antibodies used for measurement. (B) Gel filtration profile of plasma from case 2 . The left $y$-axis reflects hormone levels of collected fractions (100, hereof 38-70 is illustrated) using C-terminal glucagon assay (black curve), C-terminal amidated GLP-1 assay (red curve) and N-terminal GLP-2 assay (green curve). Calibrators (stippled black line) include ${ }^{125}$ I albumin and ${ }^{22} \mathrm{Na}$. '?' Refers to a probable biologically inactive GLP-2 immunoreactive peptide moiety.

processed from proglucagon due to exclusive expression of prohormone convertase 2 (PC2) in these cells. Studies from other groups have suggested that pancreatic islet insults and pro-inflammatory cytokines, such as interleukin 6, may induce PC1 expression and GLP-1 production within $\alpha$-cells (2). Similarly, in rats, streptozotocin-induced hyperglycaemia increases PC1 expression in islet $\alpha$-cells, thereby, liberating bioactive amidated GLP-1 to exert an insulinotropic effect on $\beta$-cells (3). In the context of proglucagon-producing tumours, these observations support a hypothesis that following the acquisition of the initial somatic genomic insult promoting tumorigenesis, some glucagonomas may undergo transition from an $\alpha$-cell-like processing profile to one that resembles the enteroendocrine L-cell through induction of tumoural PC1 expression by factors such as metabolic stress (for example, hyperglycaemia), cytokines released as part of the host immune response to malignancy and/or further somatic mutations within the tumour itself. Clinically, this might manifest as GLP-1 mediated hyperinsulinaemic hypoglycaemia on a background of a previous history of glucagonoma or diabetes mellitus. Although, we were unable to ascertain whether diabetes preceded hyperinsulinism in our first case, Roberts et al. (4) reported a GLP-1 secreting metastatic pNET in a patient with previously diagnosed diabetes. Moreover, an accompanying literature review cited other cases of metastatic pNET where hypoglycaemia preceded diabetes or other features of glucagonoma (4).

GLP-1 is released by enteroendocrine L-cells in a nutrient-dependent manner to potentiate glucosestimulated insulin secretion. Through activation of GLP-1R expressed on pancreatic islets, GLP-1 may also stimulate $\beta$-cell differentiation and proliferation, thereby, increasing $\beta$-cell mass. Recognition of the glucoselowering properties of GLP-1 has led to the development of incretin-based drugs, such as exenatide and liraglutide, for the treatment of type 2 diabetes. Notably, these therapies are associated with a low risk of hypoglycaemia. In case 1, evidence for tumoural GLP-1 mediated hyperinsulinaemic hypoglycaemia is provided by markedly elevated fasting plasma levels of bioactive GLP-1 during hypoglycaemia, absence of insulin staining in tumour biopsies and attenuation of GLP-1 together with abolition of hypoglycaemia following SSA therapy. However, the high proinsulin levels also raise the suspicion that some insulin may also derive from the tumour or some of its metastases. Unfortunately, normal pancreatic tissue was not available for further analyses to assess for islet cell hyperplasia as has been described for other metastatic GLP-1 secreting pNETs or some cases of hypoglycaemia after gastric bypass operations with high GLP-1 levels. Moreover, although no insulin staining was observed following biopsy of a single hepatic metastasis we are unable to exclude the absence of insulin production by other metastases. Thus, in light of the available data the tumour described in case 1 may be more aptly referred to as a mixed GLP-1oma and proinsulinoma.

In addition to glucose homeostasis, PGDPs are important regulators of gastrointestinal physiology and altered gastrointestinal function has been reported in other patients with enteroglucagon-secreting tumours (5). Gleeson et al. (5) first described a patient with intestinal immotility, reduced absorptive capacity and giant duodenal villi due to an enteroglucagon-producing renal 
tumour. Subsequent reports have described patients with GLP-1 and GLP-2 secreting NETs presented with intractable constipation due to intestinal immotility and gross morphological abnormalities of the small intestine identified radiologically (6) (7). Notably, none of these patients were reported to suffer from hypoglycaemia. Owing to patient refusal and/or absence of clinical indication, gastrointestinal investigations including gastroscopy and barium follow through were not undertaken in the present case; however, given symptomatic and biochemical similarities between our case and those described previously, we would anticipate similar radiological findings including giant villi, thickened mucosal folds and reduced gastric emptying.

GLP-1 is a potent inhibitor of gastric motility compared with GLP-2 whereas the latter is involved in adaptive responses to gut injury and nutrient absorption through activation of crypt cell proliferation and consequential expansion of small bowel epithelium (8). Clinically, s.c. administration of GLP-2 has been used in treatment of humans with short bowel syndrome and intestinal failure (8). Consistent with this, GLP-2 therapy increases fasting plasma citrulline concentrations, a nonamino acid biomarker of functional enterocyte mass used as a marker of attenuated absorption by enterocytes in states of intestinal failure. Interestingly, despite radiological evidence of GLP-2 intestinotrophic activity, fasting plasma citrulline concentrations were not elevated in case 1. Although, the precise explanation for this discordance remains unclear, detectable plasma citrulline negatively correlates with inflammatory cytokines and C-reactive protein and, therefore, may not under certain conditions, such as malignancy, be a reliable indicator of enterocyte mass (9). Whilst gastrointestinal dysfunction described in our first case appears to be associated with elevated levels of GLP-1 and GLP-2, we cannot dismiss the potential contribution of PYY, pancreatic polypeptide and/or other unidentified tumour-derived hormones.

Hyperglucagonaemia was a biochemical feature of both cases. In case 1, however, the majority of total circulating glucagon consisted of $\mathrm{N}$-terminal elongated glucagon moieties with subsequent analysis using a sandwich ELISA confirming that the majority of detectable glucagon comprised of incompletely processed proglucagon intermediates (Fig. 3A and B). In contrast, case 2 had 'true' hyperglucagonemia, manifesting clinically as diabetes mellitus, NME and significant weight loss. Although, the aetiology of NME remains unclear, histological similarities with pellagra (nicotinic acid deficiency) and acrodermatitis enteropathica (zinc deficiency) suggest
NME is a consequence of nutrient deficiency. Indeed, our first case had florid NME in association with low zinc levels and both cases exhibited other dermatological manifestations suggestive of nutrient deficiency. However, iatrogenic NME is also observed following i.v. glucagon infusion suggesting that PGDPs may also have direct effects on the skin (10).

In conclusion, through detailed biochemical characterisation of PGDPs secreted from proglucagonexpressing tumours we have shown that the variable clinical presentation associated with these tumours is due, at least in part, to differential processing of proglucagon and report for the first time a proglucagon-expressing tumour manifesting clinically with both symptomatic hyperinsulinism and gastrointestinal dysfunction.

Declaration of interest

The authors declare that there is no conflict of interest that could be perceived as prejudicing the impartiality of the research reported.

\section{Funding}

This research did not receive any specific grant from any funding agency in the public, commercial or not-for-profit sector.

Patient consent

Patients deceased.

\section{Author contribution statement}

B G Challis, V Bansiya, S O'Rahilly and H L Simpson cared for the patients. N J W Albrechtsen, F Gribble, K Burling, P Barker, B Hartmann and J J Holst advised and performed biochemical analyses. B G Challis, N J W Albrechtsen, J J Holst and H L Simpson prepared the manuscript.

\section{References}

1 Holst JJ 1983 Molecular heterogeneity of glucagon in normal subjects and in patients with glucagon-producing tumours. Diabetologia $\mathbf{2 4}$ 359-365. (doi:10.1007/BF00251825)

2 Ellingsgaard H, Hauselmann I, Schuler B, Habib AM, Baggio LL, Meier DT, Eppler E, Bouzakri K, Wueest S, Muller YD et al. 2011 Interleukin-6 enhances insulin secretion by increasing glucagon-like peptide-1 secretion from L cells and $\alpha$ cells. Nature Medicine 17 1481-1489. (doi:10.1038/nm.2513)

3 Nie Y, Nakashima M, Brubaker PL, Li Q-L, Perfetti R, Jansen E, Zambre Y, Pipeleers D \& Friedman TC 2000 Regulation of pancreatic PC1 and PC2 associated with increased glucagon-like peptide 1 in diabetic rats. Journal of Clinical Investigation 105 955-965. (doi:10.1172/JCI7456)

4 Roberts RE, Zhao M, Whitelaw BC, Ramage J, Diaz-Cano S, Roux CWI Quaglia A, Huang GC \& Aylwin SJB 2012 GLP-1 and glucagon secretion from a pancreatic neuroendocrine tumor causing diabetes and 
Endocrinology,

Diabetes \& Metabolism

CASE REPORT
B G Challis, N J W Albrechtsen and others
Phenotypic heterogeneity of glucagonomas hyperinsulinemic hypoglycemia. Journal of Clinical Endocrinology and Metabolism 97 3039-3045. (doi:10.1210/jc.2011-2005)

5 Gleeson MH, Bloom SR, Polak JM, Henry K \& Dowling RH 1971 Endocrine tumour in kidney affecting small bowel structure, motility, and absorptive function. Gut 12 773-782. (doi:10.1136/gut. 12.10.773)

6 Jones B, Fishman EK, Bayless TM \& Siegelman SS 1983 Villous hypertrophy of the small bowel in a patient with glucagonoma. Journal of Computer Assisted Tomography 7 334-337. (doi:10.1097/ 00004728-198306000-00009)

7 Brubaker PL, Drucker DJ, Asa SL, Swallow C, Redston M \& Greenberg GR 2002 Prolonged gastrointestinal transit in a patient with a glucagon-like peptide (GLP)-1- and -2-producing neuroendocrine tumor. Journal of
Clinical Endocrinology and Metabolism 87 3078-3083. (doi:10.1210/jcem. 87.7.8584)

8 Drucker DJ \& Yusta B 2014 Physiology and pharmacology of the enteroendocrine hormone glucagon-like peptide-2. Annual Review of Physiology 76 561-583. (doi:10.1146/annurev-physiol-021113170317)

9 Crenn P, Messing B \& Cynober L 2008 Citrulline as a biomarker of intestinal failure due to enterocyte mass reduction. Clinical Nutrition $\mathbf{2 7}$ 328-339. (doi:10.1016/j.clnu.2008.02.005)

10 Case CC \& Vassilopoulou-Sellin R 2003 Reproduction of features of the glucagonoma syndrome with continuous intravenous glucagon infusion as therapy for tumor-induced hypoglycemia. Endocrine Practice 9 22-25. (doi:10.4158/EP.9.1.22)

Received in final form 16 November 2015

Accepted 1 December 2015 